\title{
Mucosotomy at the top of the septum facilitates tunneling and clipping during peroral endoscopic myotomy for Zenker's diverticulum (Z-POEM)
}

\section{Georgios Mavrogenis ${ }^{a}$, loannis Tsevgasa ${ }^{a}$ Dimitrios Zachariadisa , Fateh Bazerbachi ${ }^{b}$}

Mediterraneo Hospital, Athens, Greece; Interventional Endoscopy Program, Massachusetts General Hospital, Boston, MA, USA

Peroral endoscopic myotomy for Zenker's diverticulum (Z-POEM) has been recently established as an effective method for complete septal dissection $[1,2]$. The cardinal steps of the procedure include a mucosotomy entrance site at $1-3 \mathrm{~cm}$ proximal to the septum, submucosal tunneling towards the top of the septum, extension of the tunnel on both esophageal and diverticular sides of the septum, complete septal myotomy, and closure of the mucosotomy. However, submucosal dissection and clipping at the level of the hypopharynx can be cumbersome because of the limited working space. To overcome this technical difficulty, we apply a modified Z-POEM technique by creating a 10-12 mm mucosal orifice along the edge of the septum (Fig. 1,2), so that a direct septotomy can be performed (Z-POES). In this manner, an adequate space is provided for the deployment of large clips and the use of a therapeutic-channel gastroscope. In this approach, the integrity of the hypopharyngeal rim of the diverticulum is preserved, since it is not accessed; this may decrease the risk of upper mediastinal intrusion and contamination. While this direct septotomy method has been proposed for patients with distorted anatomy [3], we suggest that it may also be practical in conventional cases.

\section{References}

1. Li QL, Chen WF, Zhang XC, et al. Submucosal tunneling endoscopic septum division: a novel technique for treating Zenker's diverticulum. Gastroenterology 2016;151:1071-1074.

2. Yang J, Novak S, Ujiki M, et al. An international study on the use of peroral endoscopic myotomy in the management of Zenker's diverticulum. Gastrointest Endosc 2019 May 10 [Epub ahead of print]. doi: 10.1016/j.gie.2019.04.249.

Department of Gastroenterology, ${ }^{a}$ Mediterraneo Hospital, Athens, Greece (Georgios Mavrogenis, Ioannis Tsevgas, Dimitrios Zachariadis); 'Interventional Endoscopy Program, Massachusetts General Hospital, Boston, MA, USA (Fateh Bazerbachi)

\section{Conflict of Interest: None}

Correspondence to: Georgios Mavrogenis, MD, Mediterraneo Hospital, Ilias 12, 16675 Athens, Greece, e-mail: mavrogenis@gmail.com

Received 10 September 2019; accepted 22 September 2019; published online 8 October 2019

DOI: https://doi.org/10.20524/aog.2019.0424

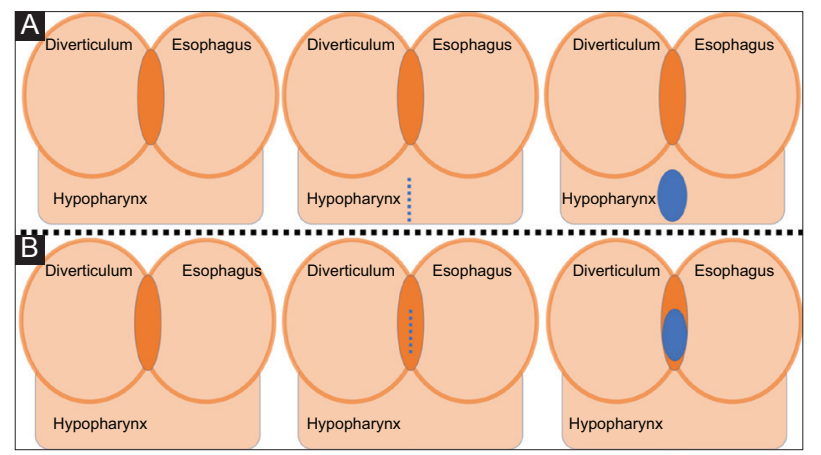

Figure 1 Schematic representation of the classic peroral endoscopic myotomy for Zenker's diverticulum (Z-POEM) and modified Z-POEM with septotomy (Z-POES). (A) In the traditional approach the mucosotomy is performed $1-3 \mathrm{~cm}$ proximally to the septum. (B) Z-POES approach with mucosotomy on the top of the septum

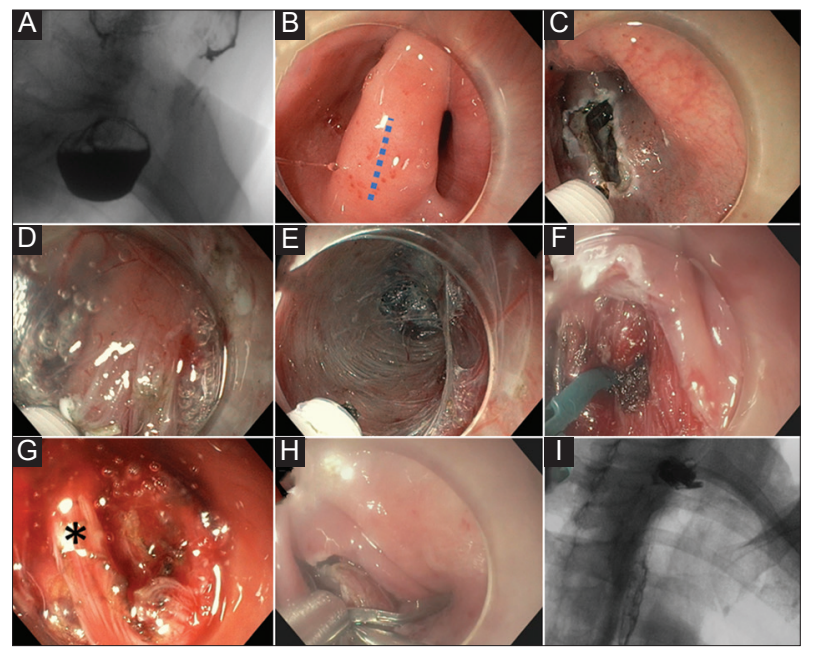

Figure 2 Clinical case of modified peroral endoscopic myotomy for Zenker's diverticulum with septotomy (Z-POES). (A) Preoperative esophagram. (B) Mucosal incision is performed on the top of the septum. (C) Mucosotomy. (D) Exposure of the septum. (E) Tunneling in the esophageal side. (F) Myotomy with a scissor-type knife. (G) The asterisk indicates the last bundle of fibers at the bottom of the septum. (H) Closure of the mucosotomy. (I) Postoperative esophagram

3. Brewer Gutierrez OI, Ichkhanian Y, Spadaccini M, Vosoughi K, Repici A, Khashab MA. Zenker's diverticulum per-oral endoscopic myotomy techniques: changing paradigms. Gastroenterology 2019;156:2134-2135. 